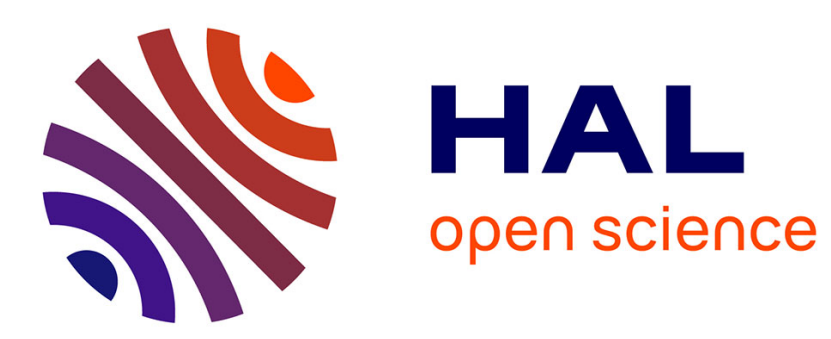

\title{
A Multi-scale Vector Spline Method for Estimating the Fluids Motion on Satellite Images
}

Till Isambert, Jean-Paul Berroir, Isabelle Herlin

\section{To cite this version:}

Till Isambert, Jean-Paul Berroir, Isabelle Herlin. A Multi-scale Vector Spline Method for Estimating the Fluids Motion on Satellite Images. European Conference on Computer Vision - ECCV 2008, Oct 2008, Marseille, France. pp.665-676, 10.1007/978-3-540-88693-8_49 . inria-00583017

\section{HAL Id: inria-00583017 https://hal.inria.fr/inria-00583017}

Submitted on 1 Jun 2011

HAL is a multi-disciplinary open access archive for the deposit and dissemination of scientific research documents, whether they are published or not. The documents may come from teaching and research institutions in France or abroad, or from public or private research centers.
L'archive ouverte pluridisciplinaire $\mathbf{H A L}$, est destinée au dépôt et à la diffusion de documents scientifiques de niveau recherche, publiés ou non, émanant des établissements d'enseignement et de recherche français ou étrangers, des laboratoires publics ou privés. 


\title{
A multi-scale vector spline method for estimating the fluids motion on satellite images
}

\author{
Till Isambert ${ }^{1,2}$, Jean-Paul Berroir ${ }^{1,2}$, and Isabelle Herlin ${ }^{1,2}$ \\ 1 INRIA, Domaine de Voluceau, Rocquencourt, BP 105 - 78153 Le Chesnay Cedex, \\ France \\ ${ }^{2}$ CEREA, Joint Laboratory ENPC - EDF R\&D, Université Paris-Est, France
}

\begin{abstract}
Satellite image sequences visualize important patterns of the atmospheric and oceanographic circulation. Assessing motion from these data thus has a strong potential for improving the performances of the forecast models. Representing a vector field by a vector spline has been proven efficient for fluid motion assessment: the vector spline formulation makes it possible to initially select the locations where the conservation equation has to be taken into account; it efficiently implements the 2nd order div-curl regularity, advocated for turbulent fluids. The scientific contribution of this article is to formulate vector splines in a multiscale scheme, with the double objective of assessing motion even in the case of large displacements and capturing the spectrum of spatial scales associated to turbulent flows. The proposed method only requires the inversion of a band matrix, which is performed by an efficient numerical scheme making the method tractable for large satellite image sequences.
\end{abstract}

\section{Introduction}

Meteorological and oceanographic forecasts are based on the numerical solving of 3D circulation models that require an initial condition of their state variables, among which velocity, i.e. winds and currents. The estimation of the initial condition relies on real measurements ingested into models by means of data assimilation [1]. Operational forecast models make use of in situ observations for this purpose, provided by ground stations, balloons, drifting buoys, etc. One challenge of environmental modelling is the ability to derive motion estimates from dynamic satellite data in order to complement in situ data. Atmospheric and oceanographic satellite images constitute a $2 \mathrm{D}$ visualization of a $3 \mathrm{D}$ fluid flow. The link between the 2D motion and the 3D flow is non trivial: for instance, on water vapor meteorological images, the pixel value results from contributions of water particles in the vertical column, hence the $2 \mathrm{D}$ image motion is a complex combination of real 3D motions. Correlation-based methods constitute the current operational standard for assessing image motion from satellite imagery (the so-called Atmospheric Motion Winds). Their applicability to environmental forecast is however limited owing to the lack of reliable tracers identifiable on images and to uncertainties affecting their height assignment. Despite these 
limitations, image motion fields are considered to be an essential data source for environmental forecast.

Assessing image motion requires defining an image processing model, constituted of a 2D conservation law and regularity properties. State-of-the-art models for fluid flow estimation are characterized by the three following properties. (1) The conservation equation applies either to the luminance [2] or to the mass [3, 4]. (2) The regularity is modelled by the 2nd order div-curl constraint, which provides direct control of the Helmholtz decomposition of the motion field in terms of divergence and vorticity [5]. A recent study [6] formulates the regularity with effective physical constraints: the retrieved flow is supposed steady and satifying the incompressible Stokes equations. The applicability to non steady flows satisfying the Navier-Stokes equations remains however open. (3) Multiscale coarse-to-fine numerical schemes [7-10] are implemented in the case of large displacements, that prevent the linearization of the conservation equation. The multiscale scheme is further needed in the case of highly turbulent flows in which motion patterns co-exist at different spatial scales.

Solving the image processing model is usually addressed by a gradient-based minimization of an energy functional made up of two components: the confidence in data and the regularity of the result. The data confidence is often the integral over the image domain of the residual of the conservation equation. Applying such an approach to satellite atmospheric or oceanographic sequences faces two main difficulties. First, the conservation equation can be locally incorrect -3D motions such as upwelling or convection constitute typical examples in which the $2 \mathrm{D}$ conservation equation is not valid- or unworkable -missing data, occlusions by clouds, absence of contrast, motion parallel to contours. Accounting for the conservation equation in the whole image domain therefore leads to erroneous flow estimates. Second, the iterative minimization of the 2nd order div-curl regularity constraint leads to 4 th order PDEs, hence to complex iterative minimization not guaranteeing the convergence to a minimum.

An elegant solution to these two problems is provided by spline-based methods. First, they make it possible to easily formulate models in which data confidence applies only at selected locations: the so-called control points. Second, they do not require an iterative energy minimization. Vector-valued thin-plate splines minimize the 2 nd order div-curl regularity constraint and have been proved efficient to interpolate or approximate wind measurements provided by ground stations [11]. The use of thin-plate vector splines for image motion estimation has been further proposed by [12], who formulated a vector spline model in which the $2 \mathrm{D}$ conservation law is satisfied at control points. Thin-plate splines are, however, unsuitable for multiscale estimation: they are defined from a harmonic radial basis function that increases with the distance to the control point and hence does not provide a local information.

This paper presents an innovative multiscale formulation of the vector spline approach for motion estimation. It keeps the nice properties of vector splines (use of control points, div-curl regularity) but makes use of a parametric spline model based on a compactly supported and rapidly decaying radial basis function, thus 
adapted to multiscale representation. The solution is obtained by solving a sparse and well-conditioned linear system. The motion is computed on a pyramidal representation of images, as the sum of a coarse scale motion and increments from one scale to the immediately finer one. Results are presented to demonstrate the effectiveness of the characteristics of the multiscale vector spline: use of control points, div-curl regularity and multiscale coarse-to-fine motion estimation.

This paper is organized as follows: the section 2 recalls the vector spline theory applied to fluid motion estimation; the proposed multiscale vector spline is presented in section 3 . Results are analyzed in section 4 , and conclusions and prospects for future work are given in section 5 .

\section{Apparent motion estimation using vector splines}

Vector splines have been initially introduced [11] for the interpolation and approximation of vector observations. In this context, the vector spline model is defined from: (1) a set of $n$ control points $\mathbf{x}_{i}$ in a spatial domain $\Omega ;(2)$ a vector observation $\mathbf{w}_{i}$ at each control point. The vector spline is solution of the following minimization problem:

$$
\begin{gathered}
\left\{\begin{array}{l}
\min \int_{\Omega}\|\mathbf{w}\|_{d}^{2} \\
\mathbf{w}\left(\mathbf{x}_{i}\right)=\mathbf{w}_{i} \forall i \\
\text { Interpolation }
\end{array} \text { or: } \min \left\{\sum_{i}\left(\mathbf{w}\left(\mathbf{x}_{i}\right)-\mathbf{w}_{i}\right)^{2}+\lambda \int_{\Omega}\|\mathbf{w}\|_{d}^{2}\right\}\right. \\
\text { Approximation }
\end{gathered}
$$

In equation (1), the parameter $\lambda$ of the approximating spline controls the compromise between regularity and confidence in data, $\|\mathbf{w}\|_{d}$ denotes the 2 nd order div-curl semi-norm, defined as:

$$
\|\mathbf{w}\|_{d}^{2}=\alpha\|\nabla \operatorname{div} \mathbf{w}\|^{2}+\beta\|\nabla \operatorname{curl} \mathbf{w}\|^{2}
$$

It is a semi-norm which zero-space is the set of affine vector fields. It has been proven [11] that this minimization problem admits a unique solution: a thin-plate spline based on the harmonic radial basis function $\phi$ :

$$
\phi(\mathbf{x})=(128 \pi)^{-1}\|\mathbf{x}\|^{4} \log \|\mathbf{x}\|
$$

The spline is defined by:

$$
\left\{\begin{array}{l}
u=p(\mathbf{x})+\sum_{i=1}^{n} a_{i}\left(\frac{1}{\alpha} \phi_{x x}\left(\mathbf{x}-\mathbf{x}_{i}\right)+\frac{1}{\beta} \phi_{y y}\left(\mathbf{x}-\mathbf{x}_{i}\right)\right)+b_{i}\left(\frac{1}{\alpha}-\frac{1}{\beta}\right) \phi_{x y}\left(\mathbf{x}-\mathbf{x}_{i}\right) \\
v=q(\mathbf{x})+\sum_{i=1}^{n} a_{i}\left(\frac{1}{\alpha}-\frac{1}{\beta}\right) \phi_{x y}\left(\mathbf{x}-\mathbf{x}_{i}\right)+b_{i}\left(\frac{1}{\alpha} \phi_{y y}\left(\mathbf{x}-\mathbf{x}_{i}\right)+\frac{1}{\beta} \phi_{x x}\left(\mathbf{x}-\mathbf{x}_{i}\right)\right)
\end{array}\right.
$$

with $p$ and $q$ degree 1 polynomials. The coefficients of the spline $\left(a_{i}, b_{i}\right.$ and the 6 coefficients in $p$ and $q$ ) are obtained by solving a linear system.

The use of vector splines for motion estimation from image data has been proposed in [12] for the luminance conservation equation, and in [13] for mass conservation. In contrast to the previous formulation, only an indirect observation is provided by the conservation equation. The latter is formulated as: 
$\mathcal{L} \mathbf{w}+I_{t}=0, \mathcal{L}$ being a linear operator. If assuming luminance conservation, we have $\mathcal{L} \mathbf{w}=\nabla I$.w, and for mass conservation: $\mathcal{L} \mathbf{w}=(\nabla I+I \nabla)$.w. For both cases, at each control point $\mathbf{x}_{i}$, we have $\mathcal{L}_{i} \cdot \mathbf{w}=-I_{t}, \mathcal{L}_{i}$ denoting the observation operator at control point $\mathbf{x}_{i}$.

The vector spline model is then rewritten as:

$$
\left\{\begin{array}{l}
\min \int_{\Omega}\|\mathbf{w}\|_{d}^{2} \\
\mathcal{L}_{i} \mathbf{w}+I_{t}=0
\end{array} \text { or: } \min \left\{\sum_{i}\left(\mathcal{L}_{i} \mathbf{w}+I_{t}\right)^{2}+\lambda \int_{\Omega}\|\mathbf{w}\|_{d}^{2}\right\}\right.
$$

for the interpolation and approximation cases. It has been proven [12] that the solution of (5) exists and is unique if the observation operators $\mathcal{L}_{i}$ are linear and non zero, and if the control points are non aligned. The solution is a thin-plate spline, with the same basis function $\phi$ as in equation (3):

$$
\mathbf{w}=\sum_{i=1}^{n} c_{i} \mathcal{L}_{i} \phi\left(\left\|\mathbf{x}-\mathbf{x}_{i}\right\|\right)+\sum_{i=1}^{6} d_{i} p_{i}(\mathbf{x})
$$

$\mathbf{p}=\left(p_{i}\right)$ being the canonical basis of degree 1 polynomials. The vectors of coefficients $\mathbf{c}=\left(c_{i}\right)$ and $\mathbf{d}=\left(d_{i}\right)$ are solution of:

$$
\left(\begin{array}{cc}
Q+\lambda I_{d} & S \\
S^{T} & 0
\end{array}\right)\left(\begin{array}{l}
\mathbf{c} \\
\mathbf{d}
\end{array}\right)=\left(\begin{array}{c}
-I t \\
0
\end{array}\right)
$$

$I_{d}$ being the identity matrix. The general term of $Q($ size $n \times n)$ is $\mathcal{L}_{i} \mathcal{L}_{j} \phi\left(\| \mathbf{x}_{i}-\right.$ $\left.\mathbf{x}_{j} \|\right)$ and $S$ (size $\left.n \times 6\right)$ is defined as: $S=\mathcal{L}_{i} \mathbf{p}$.

The vector spline model used for image motion estimation shares the same interesting properties as the model used for interpolating or approximating vector data: its solution is obtained without iterative minimization and it has few parameters (i.e. $\alpha, \beta$ and $\lambda$ ). One will set $\alpha \gg \beta$ when processing images of highly turbulent flows, thus favoring an estimation with large curl variability. Reversely, $\beta \gg \alpha$ is appropriate for situations such as intense vertical activity causing locally divergent $2 \mathrm{D}$ flows.

One main difference with vector data is that there are no predefined locations where observations are available. The control points selection process must be defined prior to the estimation. The theoretical conditions guaranteeing the existence and uniqueness of the solution can easily be met by selecting these control points with a double thresholding: first on the magnitude of the spatial gradient, discarding low contrast areas; second on the magnitude of the motion index (ratio of temporal and spatial gradient), thus discarding visually still areas. Depending on the applicative domain, further locations in specific structures must be discarded. This is, for instance, the case of ejection filaments in oceanography, as in these structures the motion is parallel to the contours and the conservation equation is degenerated. The selection of control points is therefore highly application-dependent; they should be as evenly distributed as possible, but there is, to our knowledge, no criterion for defining an optimal distribution of control points. 


\section{Multiscale vector splines}

\subsection{Parametric spline model}

Thin-plate vector splines minimize the 2nd order div-curl regularity, but are inappropriate for multiscale estimation as they are defined from a harmonic basis function. A multiscale scheme actually requires using a basis function that provides a local representation, hence locally supported or rapidly decaying. The contribution of this paper is to formulate a multiscale model, based on a spline parameterized by the scale value and on a pyramidal representation of images at different scales.

We consider the spline approximation problem with the 2nd order div-curl norm and either the luminance or the mass conservation equation, through the observation operators $\mathcal{L}_{i}$ assessed on the $n$ control points $\mathbf{x}_{i}$ :

$$
\min J(\mathbf{w})=\left\{\sum_{i=1}^{n}\left(\mathcal{L}_{i} \mathbf{w}-\mathbf{w}_{i}\right)^{2}+\lambda \int_{\Omega} \alpha\|\nabla \operatorname{div} \mathbf{w}\|^{2}+\beta\|\nabla \operatorname{curl} \mathbf{w}\|^{2}\right\}
$$

Rather than exactly solving equation (8), which would lead to the thin-plate spline, the minimum is searched for among a set of spline functions suitable for the multiscale formalism and satisfying the two following properties. (1) The spline is defined from a unique bell-shaped radial basis function of unit support. The choice of this function is not critical as long as it is positive, decreasing and at least three times continuously differentiable in order to compute the 2nd order div-curl semi-norm. We make use of the basis function $\psi$ proposed by [14] and defined as $\psi(r)=(1-r)^{6}\left(35 r^{2}+18 r+3\right)$ for $|r| \leq 1$. (2) The spline is a linear combination of translates of the basis function over a regular lattice of $m$ grid points, whose sampling defines the scale parameter $h$. These translates are dilated by a factor $\gamma$ proportional to $h$. The parameters defining the spline are the $m$ weights $\mathbf{q}=\left(\mathbf{q}_{j}\right)$ (each weight $\mathbf{q}_{j}$ being homogeneous to a motion vector with $u$ and $v$ components) applied to the translates of the basis function. The parametric expression of the vector spline is thus:

$$
\mathbf{w}_{\mathbf{q}, h}(x)=\sum_{\mathbf{v}_{j} \in \mathbb{Z}^{2}, h \mathbf{v}_{j} \in \Omega} \mathbf{q}_{j} \psi\left(\left\|\frac{\mathbf{x}-h \mathbf{v}_{j}}{\gamma}\right\|\right)
$$

where $\mathbf{v}_{j}$ spans a regular lattice of unit spacing in the image domain $\Omega$.

A new expression of the functional $J$ is defined by substituting, in equation (8), w by its parametric form $\mathbf{w}_{\mathbf{q}, h}(9)$. Let us first consider the first term of $J$. If the observation operator is based on the luminance conservation equation, its new expression becomes:

$$
\left\|\mathbf{I}_{\mathbf{x}} \Psi \mathbf{q}^{\mathbf{u}}+\mathbf{I}_{\mathbf{y}} \Psi \mathbf{q}^{\mathbf{v}}-\mathbf{I}_{\mathbf{t}}\right\|^{2}=\left\|A_{l} \mathbf{q}-\mathbf{I}_{\mathbf{t}}\right\|^{2}
$$

$\mathbf{I}_{\mathbf{t}}$ being the $n$-dimensional vector of the temporal derivatives at the control points; $\Psi$ being the $n \times m$ matrix of general term $\psi\left(\left(x_{i}-k_{j}\right) / \gamma,\left(y_{i}-l_{j}\right) / \gamma\right)$ with $i$ indexing the $n$ control points and $j$ the $m$ grid points $(k, l) ; \mathbf{I}_{\mathbf{x}}$ and $\mathbf{I}_{\mathbf{y}}$ are the 
$n \times n$ diagonal matrices of the image spatial derivatives at the control points. In the case of mass conservation, the first term of $J$ becomes:

$$
\left\|\mathbf{I}_{\mathbf{x}} \Psi \mathbf{q}^{\mathbf{u}}+\mathbf{I}_{\mathbf{y}} \Psi \mathbf{q}^{\mathbf{v}}+\mathbf{I D}_{\mathbf{x}} \Psi+\mathbf{I D}_{\mathbf{y}} \Psi-\mathbf{I}_{\mathbf{t}}\right\|^{2}=\left\|A_{m} \mathbf{q}-\mathbf{I}_{\mathbf{t}}\right\|^{2}
$$

where $\mathbf{I}$ is the $n \times n$ diagonal matrix formed by the image values at control points, $\mathbf{D}_{\mathbf{x}} \Psi$ and $\mathbf{D}_{\mathbf{y}} \Psi$ are the matrices of the spatial derivatives of $\Psi$. Whatever the conservation equation, the first term of $J$ is then rewritten as a quadratic function of $\mathbf{q}$.

Let us now analyze the second term of $J$. By introducing the matrix of differential operators $Q(D)$ :

$$
Q(D)=\left(\begin{array}{cc}
\sqrt{\alpha} \partial_{x x}+\sqrt{\beta} \partial_{y y} & (\sqrt{\alpha}-\sqrt{\beta}) \partial_{x y} \\
(\sqrt{\alpha}-\sqrt{\beta}) \partial_{x y} & \sqrt{\alpha} \partial_{y y}+\sqrt{\beta} \partial_{x x}
\end{array}\right)
$$

$J$ then factorizes as:

$$
\alpha \int\|\nabla \operatorname{div} \mathbf{w}\|^{2}+\beta \int\|\nabla \operatorname{curl} \mathbf{w}\|^{2}=\int\|Q(D) \mathbf{w}\|^{2}
$$

The second term of $J$ is finally rewritten as the quadratic expression $\|R \mathbf{q}\|^{2}$, with:

$$
R=\left(\begin{array}{cc}
\sqrt{\alpha} \partial_{x x} \Psi+\sqrt{\beta} \partial_{y y} \Psi & (\sqrt{\alpha}-\sqrt{\beta}) \partial_{x y} \Psi \\
(\sqrt{\alpha}-\sqrt{\beta}) \partial_{x y} \Psi & \sqrt{\alpha} \partial_{y y}+\sqrt{\beta} \partial_{x x} \Psi
\end{array}\right) .
$$

The substitution of $\mathbf{w}$ by the parametric expression $\mathbf{w}_{\mathbf{q}, h}$ allows $J$ to be rewritten as a quadratic function of $\mathbf{q}$ :

$$
J(\mathbf{q})=\left\|A \mathbf{q}-I_{t}\right\|^{2}+\lambda\|R \mathbf{q}\|^{2}
$$

with $A$ being either $A_{l}$ or $A_{m}$ depending on the conservation equation chosen. Finding the minimum of $J$ with respect to $\mathbf{q}$ is now a linear optimization problem.

The matrices $A$ and $R$, in (15), have a band structure since $\psi$ has a compact support of size $\gamma$. The width of the band depends on the ratio of $\gamma$ to the scale parameter $h$. If $\gamma$ is smaller than $h$, the matrices $A$ and $R$ are diagonal and the vector spline is zero everywhere except in the vicinity of the grid points. If $\gamma$ is large compared to $h$, the resulting vector spline can accurately approximate the thin-plate spline, but the $A$ and $R$ matrices are dense and require a heavy computational load. $\gamma=3 h$ has been empirically chosen as a good compromise between the computational speed and the accuracy of the spline. The band structure allows an efficient numerical solving to be implemented.

\subsection{Hierarchical motion estimation}

A multiscale scheme is required for two main reasons. (1) The parametric spline allows the image motion to be assessed, given a spatial scale parameter $h$, and provided that the conservation equation can be computed. On satellite image 
sequences, a too strong motion and/or a too coarse time sampling cause large displacements between successive frames, preventing the linearization of the conservation equation. (2) Turbulent flows are associated with a large spectrum of spatial and temporal scales. We therefore make use of a pyramidal scheme, in which motion is hierachically computed from the coarsest to the finest scale.

Let $I_{0}$ and $I_{1}$ be two successive images of the sequence. Both are represented using a pyramid, from the full resolution $I_{0}(0)$ and $I_{1}(0)$ to the coarsest scale $I_{0}\left(p_{\max }\right)$ and $I_{1}\left(p_{\max }\right)$. To each index $p$ corresponds a scale parameter $h(p)$. The motion is initially computed at the coarsest scale with the parametric spline at scale $h\left(p_{\max }\right)$, yielding the motion field $\mathbf{w}\left(p_{\max }\right)$. This initial coarse motion field is then progresively refined at each scale $h(p)$ by first compensating the image $I_{0}(p)$ with $\mathbf{w}(p+1)$ and computing the motion increment $\delta \mathbf{w}(p)$ between the compensated image and $I_{1}(p)$. The finest scale motion $(p=0)$ is thus expressed as the sum of the coarse scale motion $\mathbf{w}\left(p_{\max }\right)$ and of the increments describing the finer resolutions:

$$
\mathbf{w}(0)=\mathbf{w}\left(p_{\max }\right)+\sum_{p=p_{\max }-1}^{0} \delta \mathbf{w}(p)
$$

The link between the scale parameter $h(p)$ and the real spatial scale of the evolving image structures is not obvious: at one level of the pyramid, the motion is computed using a scale parameter $h(p)$ corresponding to a basis function of support $\gamma=3 h(p)$. The basis function is thus able to represent motion patterns with spatial size less than $3 h(p)$; but there is no guarantee that all motion patterns of that size will be represented: this will occur only if enough control points have been selected in the existing patterns.

\section{Results}
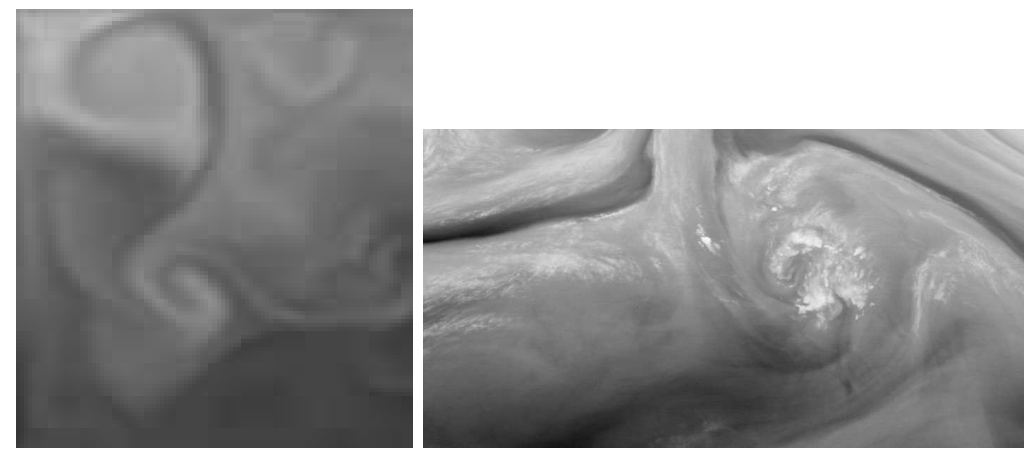

Fig. 1. Extract of the test sequences: left, OPA; right: Meteosat. 
The first result intends to demonstrate the efficiency of accounting for the conservation only at control points. For this purpose, the motion is computed using the multiscale vector spline and compared to the result of Corpetti's method [15]. Both methods minimize the second order div-curl regularity constraint, make use of either luminance or mass conservation and are solved in a multiscale scheme. The two methods differ in the data confidence term of the minimized energy (computed on control points selected by double thresholding for the multiscale spline, on the whole image domain for Corpetti's method) and in the numerical minimization scheme (multiscale vector spline vs variational minimization). Two comparisons are displayed. First, the motion is computed using the luminance conservation equation on the synthetic 'OPA' sequence (on the left in figure 1), obtained by numerical simulation with the OPA ocean circulation model ${ }^{3}$. The OPA sequence consists of simulated images of sea surface temperature, used for computing motion. Additionaly the corresponding surface currents are available and used as the reference field for validation purposes. The results are displayed on figure 2. The mean angular error between the estimated and reference motion fields is 28 degrees for the multiscale spline and 42 degrees for Corpetti's method. The qualitative inspection of the motion field's streamlines and vorticity suggest that the motion of vortices is better assessed by the multiscale spline. A similar comparison on a Meteosat- 5 sequence $^{4}$ acquired in the water vapor band is displayed on figure 3 . The mass conservation equation is used as the $2 \mathrm{D}$ atmospheric flow can be considered as compressible to accomodate the effects of vertical motion. For this sequence, a sole qualitative assessment of results is possible. The multiscale spline is more accurate with respect to the location of the central vortex. It furthermore succeeds in capturing a rotating motion in the lower left part of the image, whereas Corpetti's method incorrectly computes a smooth laminar field.

The second comparison is intended to demonstrate that the 2nd order div-curl regularity must be preferred to $L^{2}$ regularity for fluid motion assessment. The luminance conservation equation is considered and the motion is computed on the OPA sequence by the multiscale spline and the Horn and Schunck method [2]. The results are displayed on figure 4 . Three different results are presented corresponding to different values of the $\lambda$ coefficient assigned to the regularity component, so that both methods are tested with low, medium and high regularization. The angular errors for the multiscale spline are 30, 29 and 28 degrees (respectively for low, medium and high regularity), for the Horn and Schunk method 43, 47 and 49 degrees. The spline method is much more efficient as far as the detected location of eddies is concerned: only one vortex is detected by H\&S method with low regularity, and none with medium and high regularity. This is a consequence of the $L^{2}$ regularization which favours laminar fields.

Figure 5 displays the motion fields estimated on the OPA sequence at three different scales. At the coarsest scale, the main vortices appear in the upper part of the image, and the large vortex in the bottom part is not detected at all. At

\footnotetext{
3 Thanks to Marina Levy, LOCEAN, IPSL, France

${ }^{4}$ Copyright Eumetsat
} 


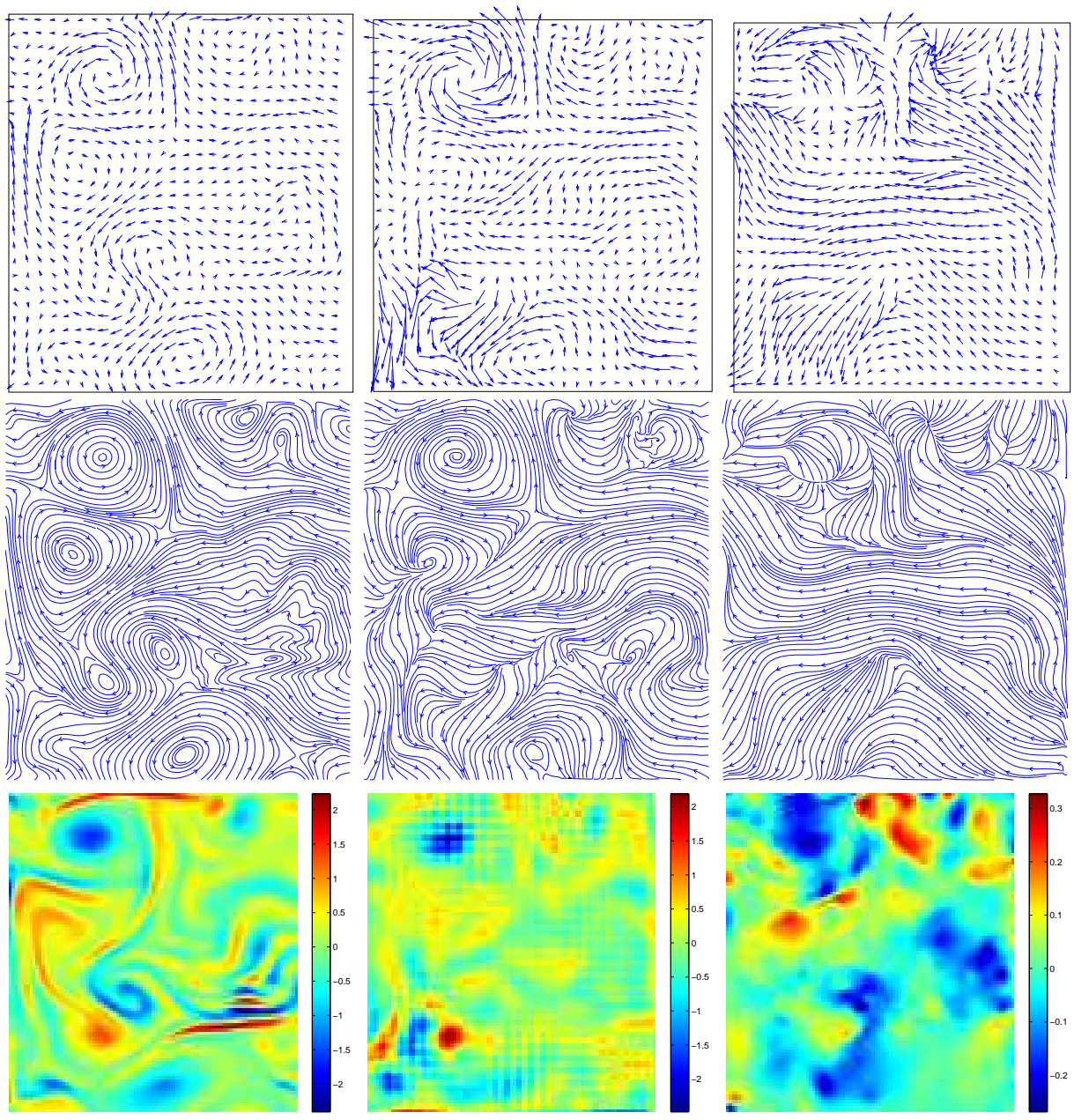

Fig. 2. Motion fields estimated on the OPA sequence using luminance conservation. Left to right: reference motion, multiscale spline, Corpetti and Mémin. Top to bottom: motion field, streamlines, vorticity. 

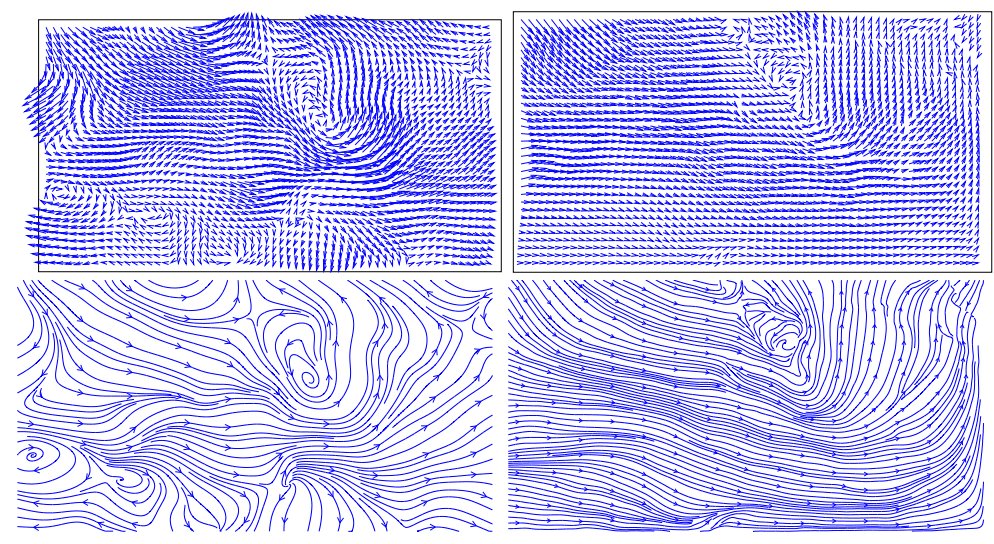

Fig. 3. Motion fields estimated on the Meteosat sequence using mass conservation. Left: multiscale spline, right: Corpetti and Mémin. Top to bottom: motion field, streamlines.

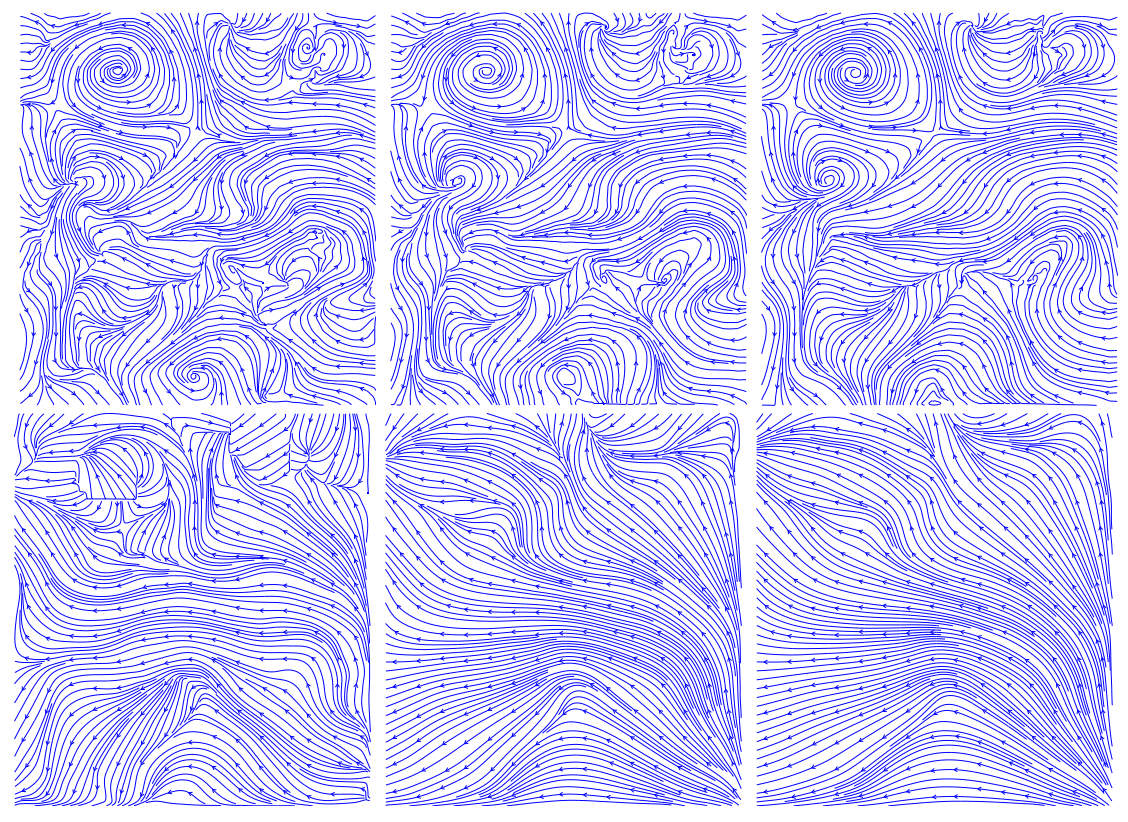

Fig. 4. Comparison of motion estimates on the OPA sequence, luminance conservation. Top: multiscale spline with low, medium and high regularity; bottom: Horn and Schunk with low, medium and high regularity. 
the intermediate scale, more vortices appear. At finest resolution the location of vortices is improved and the large vortex in the bottom part of the image is even detected. This illustrates that the multiscale scheme actually links the size of the spatial structure with the spatial scale of the spline, although this link is not easy to interpret.

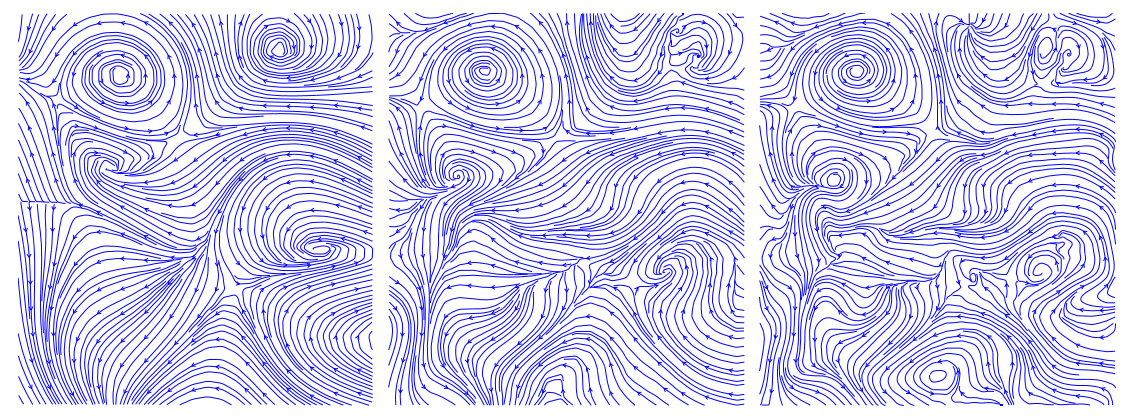

Fig. 5. Motion field estimated on the OPA sequence, from the coarsest to the finest (full) resolution.

\section{Conclusion and future work}

This paper proposes an innovative approach for assessing motion on atmospheric and oceanographic satellite image sequences of fluid flows. Its characteristics are the following. (1) The data confidence is only taken into account at specific locations, the control points, where the conservation equation is workable, i.e. valid in $2 \mathrm{D}$, or not degenerated to $0=0$. The comparison made with a method using the same conservation equation and the same regularity constraint, but computing the conservation equation over the whole image domain, proves that using control points is essential for assessing the confidence in data. (2) The regularity of the motion field is controlled by the 2nd order div-curl constraint. This is especially adapted to fluid and turbulent flows, as it allows the variations of divergence and vorticity of the retrieved motion field to be controlled. This is highlighted by comparisons with methods based on $L^{2}$ regularization, which are unable to correctly assess the motion field in eddies. (3) A multiscale scheme is used to to allow estimation even in the case of large displacements and to provide a hierarchical representation of the motion field, distinguishing between large and fine scale structures such as vortices.

Being based on control points and on the 2nd order div curl constraint, the motion field should theoretically be expressed by a thin-plate spline. The theoretical contribution of this paper is to formulate a vector spline in a multiscale scheme, using a parametric spline model. This approach has two main advantages: (1) the minimum of the energy is obtained by solving a linear system; (2) 
the matrix to invert has a band structure, allowing an efficient implementation that makes the method affordable even for large sequences.

The approach requires further investigation on two issues. First, what is the link between the spatial scale of basis functions and the extent of image structures such as vortices? There is no obvious link according to the results and we believe that the control points must also be hierarchically organized for that purpose. Second, it is well known that spatial and temporal scales of turbulent flows are strongly linked, large vortices being stabler than smaller ones. It is thus necessary to consider motion estimation not only from a pair of successive images, but from a longer sequence in order to establish a multiscale representation both in space and time.

\section{References}

1. Korotaev, G., Huot, E., Le Dimet, F.X., Herlin, I., Stanichny, S., Solovyev, D., Wu, L.: Retrieving Ocean Surface Current by 4D Variational Assimilation of Sea Surface Temperature Images. Remote Sensing of Environment (2007) Special Issue on Data Assimilation.

2. Horn, B., Schunck, B.: Determining optical flow. AI 17(1-3) (August 1981) 185-203

3. Béréziat, D., Herlin, I., Younes, L.: A generalized optical flow constraint and its physical interpretation. In: CVPR'00. (2000) 487-492

4. Wildes, R., Amabile, M.: Physically based fluid flow recovery from image sequences. In: CVPR'97, Puerto Rico (June 1997) 969-975

5. Gupta, S., Princ, J.: Stochastic models for div-curl optical flow methods. IEEE Signal Processing Letter 3(2) (1996)

6. Ruhnau, P., Schnoerr, C.: Optical Stokes Flow Estimation: An Imaging-based Control Approach. Experiments in Fluids 42 (2007) 61-78

7. Anandan, P.: A computational framework and an algorithm for the measurement of visual motion. International Journal of Computer Vision 2 (1989) 283-310

8. Bergen, J., Anandan, P., K.J., H., Hingorani, R.: Hierarchical model-based motion estimation. In: ECCV '92, London, UK (1992) 237-252

9. Enkelmann, W.: Investigation of multigrid algorithms for the estimation of optical flow fields in image sequences. Computer Vision Graphics and Image Processing 43(2) (August 1988) 150-177

10. Moulin, P., Krishnamurthy, R., Woods, J.: Multiscale modeling and estimation of motion fields for video coding (1997)

11. Amodei, L.: A vector spline approximation. Journal of approximation theory 67 (1991) 51-79

12. Suter, D.: Motion estimation and vector splines. In: CVPR94. (1994)

13. Isambert, T., Herlin, I., Berroir, J., Huot, E.: Apparent motion estimation for turbulent flows with vector spline interpolation. In: XVII IMACS, Scientific Computation Applied Mathematics and Simulation, Paris, July, 11-15. (2005)

14. Wendland, H.: Piecewise polynomial, positive definite and compactly supported radial basis functions of minimal degree. Advances in Computational Mathematics 4 (1995) 389-396

15. Corpetti, T., Memin, E., Perez, P.: Dense estimation of fluid flows. PAMI 24(3) (March 2002) 365-380 